Sánchez Castro, N. \& Facal, D. (2018). Las relaciones entre las familias y la escuela en la etapa adolescente: implicaciones para el Departamento de Orientación. Revista Electrónica Interuniversitaria de Formación del Profesorado, 21(3), 63-74.

\title{
Las relaciones entre las familias y la escuela en la etapa adolescente: implicaciones para el Departamento de Orientación
}

\author{
Noelia Sánchez Castro, David Facal \\ Departamento de Psicoloxía Evolutiva e da Educación, Universidade de Santiago de \\ Compostela.
}

\section{Resumen}

El desarrollo del alumnado adolescente tiene lugar en una sociedad en constante cambio y se ve influenciado por factores económicos, sociales y culturales que es necesario tener en cuenta desde la institución escolar para mejorar nuestras intervenciones. La presente investigación documental tiene por objetivo realizar un análisis de las nuevas tendencias en el estudio del desarrollo adolescente, prestando especial atención a las relaciones entre las familias y los centros de educación secundaria. Para ello se realizó una búsqueda bibliográfica en las bases de datos PsycInfo, Web of Science y Eric.

La tendencia que se observa en las relaciones entre las familias de los adolescentes y los centros de secundaria es una disminución en la participación y en la implicación de las primeras en las actividades escolares de sus hijos a medida que estos avanzan de curso. Además, estas relaciones presentan un marcado carácter bidireccional, están vinculadas a situaciones negativas y, generalmente, son iniciadas por los docentes, sirviendo a una función informativa más que participativa.

Teniendo en cuenta esta realidad, se sugieren acciones que pueden ser llevadas a cabo desde los Departamentos de Orientación de los centros educativos para mejorar las relaciones entre estos dos colectivos y hacer de la escuela un espacio abierto a toda la comunidad.

\section{Palabras clave}

Participación familiar; adolescencia; orientación; relación familia-escuela.

\section{Contacto:}

David Facal, david.facal@usc.es, Facultade de Psicoloxía - Rúa Xosé María Suárez Núñez, s/n. Campus vida.15782 Santiago de Compostela. 


\title{
Relationships between families and school in the teenager stage: implications for the Guidance Department
}

\begin{abstract}
The development of adolescent students takes place in a society in constant change and is influenced by economic, social and cultural factors that must be taken into account from the school institution to improve our interventions. The present documentary research aims to perform an analysis of new trends in the study of adolescent development, paying special attention to the relationships between families and schools. To do this, a bibliographic search was carried out in the PsycInfo, Web of Science and Eric databases.
\end{abstract}

The tendency observed in the relations between the families of the adolescents and the school is a decrease in the participation and in the involvement of the former in the school activities of their children as they progress through the year. In addition, these relations have a marked bi-directional character, are linked to negative situations and, generally, are initiated by teachers, serving an informative rather than participative function.

Taking into account this reality, we suggest actions that can be carried out from the Guidance Departments of the educational centers to improve the relations between these two groups and make the school a space open to the whole community.

\section{Key words}

Family participation; adolescence; orientation; family-school relationship

\section{Introducción}

La entrada en el siglo XXI se asocia con la introducción de grandes cambios que afectan a toda la sociedad en todos sus niveles. Concretamente, se producen cambios a nivel político, social, económico y cultural, que consolidan un mundo caracterizado por la globalización, los movimientos migratorios, los cambios demográficos, y el uso de nuevas tecnologías, así como un individualismo creciente y la duda o incertidumbre constante.

Si el mundo está en constante cambio, también lo estará la educación, la familia y la adolescencia, entendida como una etapa del ciclo vital culturalmente construida. Por lo tanto, desde los Departamentos de Orientación de los centros de educación secundaria es importante conocer las características que definen actualmente la sociedad y cómo afectan a los colectivos con que estos trabajan, es decir, familias y adolescentes, para así poder favorecer su desarrollo.

Para analizar el impacto que tiene el contexto socio-histórico actual en las adolescencia, resulta útil el modelo ecológico, el cual propone los factores macrosistémicos pueden influir sobre lo que ocurre en los contextos más próximos a los adolescentes (Oliva, 2003). Una pregunta clave es si esas influencias facilitan el desarrollo o si por el contrario suponen nuevos retos y dificultades. Dichas influencias dan lugar a un adelanto gradual en la edad de inicio de la pubertad debido, en parte, a las mejoras en la calidad de vida. Esto nos lleva a encontrarnos con niños que todavía muestran una importante inmadurez psicológica pero que, sin embargo, muestran madurez física. Además, muchos de los comportamientos que 
antes eran frecuentes en edades más avanzadas de la adolescencia comienzan a manifestarse en la niñez tardía, como por ejemplo, las relaciones de pareja o el consumo de ciertas substancias (Oliva, 2006). También se observa un retraso en la edad de inicio de la etapa laboral en los adultos más jóvenes y en la emancipación de los mismos, hecho que podría estar justificado por la dificultades para encontrar empleo o las buenas relaciones con los padres (Arnett, 2008).

Los cambios sociales que se producen con la llegada del nuevo siglo también afectan a las familias (Oliva, 2006). Las familias son cada vez menos numerosas en los países desarrollados, y atrás quedó el modelo de familia autoritaria, dando paso a un estilo mucho más democrático e igualitario. Esto último tiene una influencia positiva en la autoestima y satisfacción de los adolescentes, la cual se puede volver negativa cuando nos encontramos con padres excesivamente permisivos o incluso indiferentes.

Por otra parte, en las últimas décadas se han producido cambios en la estructura familiar tradicional, dando lugar a una gran variedad de modelos de familia. El número de separaciones aumentó en los últimos años, igual que el número de hijos nacidos fuera del matrimonio. Es decir, cada vez son más frecuentes las familias monoparentales y reconstituidas. Estos cambios pueden estar determinados, entre otros factores, por la libertad de los adultos para vincularse y desvincularse sin necesidad de formar una pareja para siempre o por el mayor individualismo, aumentando así el valor concedido al propio currículo y dejando en un segundo plano la familia y los hijos (Elzo, 2006).

Si analizar la relación entre los adolescentes y sus familias es importante para poder optimizar su desarrollo, también lo es analizar las relaciones que se dan entre esas familias y los centros educativos, ya que como se dijo en líneas precedentes, familia y escuela son los principales contextos de socialización de niños y adolescentes.

Sin embargo, resulta paradójico que a pesar de que tanto profesores como familias comparten un mismo objetivo respecto a los adolescentes (favorecer su desarrollo y su aprendizaje, preparándolos para ser adultos responsables), la realidad muestra que la colaboración entre ambos colectivos en su tarea educativa es escasa; y más concretamente, a medida que se avanza en el sistema educativo, la intensidad y frecuencia de la participación escolar por parte de las familias disminuye. Existen distintas formas de colaboración como el Consejo Escolar, la AMPA, o la tutoría pero sin embargo, o no se emplean o se basan fundamentalmente en metodologías comunicativas e informativas de carácter unidireccional: desde el profesorado a las familias.

Si echamos la vista atrás podemos identificar como la escuela tradicionalmente tenía por función instruir y transmitir conocimientos al alumnado mientras que la familia era la encargada de la formación de carácter más personal y afectivo. Sin embargo, en la actualidad las familias sobrevaloran las posibilidades de educación escolar y delegan en el profesorado ciertas funciones relacionadas con la formación en valores o la construcción de la identidad, bajo la creencia de que los docentes educarán mejor a sus hijos. Ante este panorama, la escuela es insuficiente para cubrir todas esas funciones y el profesorado en muchas ocasiones se siente incapaz de llevarlas a cabo.

De este modo, surge la necesidad de fortalecer la colaboración entre la familia y la escuela en la educación del alumnado adolescente ya que, tal y como diversas investigaciones manifiestan, esto repercute en una mejora en el rendimiento escolar de estos últimos, así como en el desarrollo de actitudes y comportamientos positivos (Siles, 2003). Es fundamental que los hijos perciban la continuidad entre los objetivos educativos de sus padres y los del centro escolar. Estas acciones, también tienen efectos positivos sobre las 
familias, ya que favorecen el desarrollo de actitudes positivas de cara al centro; y sobre el profesorado, aumentando su motivación (Hernández y López, 2006).

Por lo tanto, este trabajo tiene por objetivo realizar una revisión documental sobre la bibliografía existente en cuanto a las relaciones entre las familias de los adolescentes y la escuela y, a partir de ella, aportar una serie de implicaciones prácticas que los Departamentos de Orientación de los centros educativos deberían tener en cuenta para mejorar dichas relaciones $\mathrm{y}$, en última instancia, mejorar el desarrollo integral del adolescente.

\section{Metodología}

La presente investigación consiste en una investigación documental sobre las relaciones actuales entre familias y escuela en las bases de datos Psyclnfo, Web of Science y Eric utilizando los siguientes descriptores: "school", "adolescents", "family", "school-family relationship" y "parent-teacher collaboration". Como criterios de inclusión se establecieron los siguientes:

a) Fecha de publicación: desde el 2006 hasta el 2016, acotando así el volumen de información existente sobre el tema a los últimos diez años debido a la gran cantidad de estudios existentes sobre el mismo.

b) Idioma: castellano e inglés.

C) Tipo de publicaciones: artículos, tesis y libros.

d) Relación con el tema: relaciones familia-escuela en la actualidad.

Se obtuvieron un total de 197 resultados de los cuales, mediante una lectura de resúmenes de aquellos que contenían como palabras clave los descriptores empleados en la búsqueda, se seleccionaron 26 para una lectura de texto completo. Finalmente, 7 fueron incluidos en la revisión, comprobando que realmente sí cumplían con los criterios inicialmente estipulados (fecha de publicación desde el 2006 hasta el 2016, publicados en inglés o castellano y relacionados con el tema de las nuevas tendencias en las relaciones entre familia y escuela). Por último, también se realizó una búsqueda manual de información a partir de la bibliografía encontrada en los artículos seleccionados. De esta forma se incluyó 1 artículo más en la revisión, la cual cuenta con un total de 8 artículos analizados.

Para la presentación de los resultados, el análisis de los textos encontrados se hizo en función de los objetivos de la revisión documental y utilizando la Tabla 1 como guía para completar la información.

\section{Resultados}

A continuación se exponen, siguiendo un orden cronológico, los principales estudios que permiten dar respuesta a nuestro primer objetivo: conocer cómo son actualmente las relaciones entre las familias y la escuela y las expectativas que familias y profesorado tienen al respecto.

\section{Estado de la cuestión}

Blândul (2012), estudió el grado de establecimiento de relaciones entre escuela y familia en la educación del alumnado centrándose en la implicación de los padres en el diseño y en la realización de actividades y en la gestión del comportamiento de sus hijos. El instrumento 
empleado para realizar la investigación consistía en un cuestionario de 32 ítems. Las categorías que contenía dicho cuestionario incluían, entre otras, los métodos de colaboración entre escuela y familia, la gestión de la crisis educativa o la cooperación entre profesores en actividades didácticas y extradidácticas. Los resultados evidenciaron que la mayoría de los docentes nunca consultaban a los padres para el diseño de las tareas de enseñanza, aprendizaje y evaluación. Sorprendentemente, tampoco se pedía la colaboración de las familias para la gestión de los problemas de comportamiento que pudieran surgir en el aula. Por el contrario, los profesores si que afirmaban ofrecer asesoramiento a los padres sobre como ayudar a sus hijos a aprender. Es decir, los docentes buscan la colaboración de las familias cuando existen problemas de educación. Por lo tanto, este estudio muestra, en relación con el objetivo nuestra investigación, que en la mayoría de los casos la relación entre la familia y la escuela se da con un marcado carácter unidireccional, desde la escuela a los padres.

Kim y Hill (2015), realizaron un metaanálisis sobre el efecto de la participación de los padres en la escuela sobre el rendimiento académico del alumnado, y si existían diferencias en función del género de los progenitores y de la etapa educativa. Dicho metaanálisis incluía 52 estudios y un total de 390 correlaciones para la relación entre participación familiar y rendimiento académico. Los resultados evidenciaron que la relación entre participación y logros académicos difiere en función del tipo de participación tanto para padres como para madres. Las menores relaciones fueron obtenidas en el caso de participación en el hogar, seguido de participación académicas y a continuación socialización académica. La intensidad de la relación no mostraban diferencias entre padres y madres en el caso de participación en el hogar y socialización académica pero sí que lo hacían en la participación en la escuela, siendo mayor para las madres. Con respecto a la etapa educativa, los resultados mostraron que no existían diferencias entre la participación de padres y de madres a lo largo de las distintas etapas.

Hampden-Thompson y Galindo (2016) investigaron las influencias de las relaciones entre la familia y la escuela sobre los logros académicos de los adolescentes. Sus hipótesis de partida contemplaban la existencia de una asociación positiva entre las relaciones familiaescuela y los resultados académicos de los adolescentes, y que esta asociación estaría parcialmente mediada por la satisfacción de los padres con la escuela de sus hijos. La muestra empleada estaba compuesta por 10572 participantes, extraídos de un estudio longitudinal realizado en Inglaterra a adolescentes. El logro académico constituyó la variable dependiente y fue evaluado a través de las cualificaciones obtenidas por los adolescentes. En el primer año del estudio también se recogieron datos sobre las relaciones entre la familia y la escuela y sobre la satisfacción de los padres con la escuela, los cuales sirvieron para evaluar las otras dos variables de interés para el estudio. Los resultados evidenciaron, en primer lugar, que las relaciones familia-escuela y la satisfacción de los padres fue mayor a medida que el rendimiento académico de los sujetos también aumentó. Así, a medida que aumentaban las relaciones entre familia-escuela, o a medida que aumentaba la satisfacción de los padres con la escuela, también se incrementaban los logros académicos. Al incluir la variable satisfacción como mediadora, la asociación entre relaciones familia-escuela y resultados académicos dejó de ser significativa. Por lo tanto, la primera hipótesis se confirmó pero, por el contrario, la segunda no ya que se observó que la variable satisfacción de los padres explicaba por completo la asociación entre las relaciones familia-escuela y los logros académicos.

Dado que vivimos en un mundo en el que existe un predominio de las tecnologías de la información y de la comunicación (TIC) también hay investigaciones que se centran en analizar las relaciones entre las familias y la escuela a través de este medio. Así, nuevamente 
en el año 2016, Seoane y Sabé realizaron una investigación que trataba de analizar las relaciones digitales existentes entre docentes y familias, así como cual debería ser el rol que desempeñaran las TIC en la educación para favorecer dichas relaciones y el éxito escolar del alumnado.

La metodología de la investigación consistió en un estudio de casos múltiples mediante técnicas de investigación documental para conocer las aplicaciones empleadas en los colegios objeto de estudio, y entrevistas semiestructuradas a 10 profesores encargados del uso de esas aplicaciones. La información obtenida fue categorizada y analizada en torno a diez dimensiones, las cuales nos permiten conocer cómo son en la actualidad las relaciones entre familia y escuela, en este caso a través de las TIC, un medio tan presente en nuestro día a día. Entre los resultados destacan ideas como que las aplicaciones empleadas mayoritariamente por los centros son las webs y los blogs, las plataformas de intranet, el correo electrónico, las redes sociales y las "newsletters". La motivación del uso de las TIC en los centros era puramente comercial en la mayoría de los centros analizados ya que afirmaban (aunque no de forma explícita) que las usaba para estar al día en las innovaciones; y las funciones que se le atribuyen son informativas o comunicativas, pero en ninguno de ellos se utilizan con una función participativa. Además, los propios centros manifiestan la existencia de dificultades de acceso a las infraestructuras por parte de las familias, así como de alfabetización digital de los progenitores. Por lo tanto, con esta investigación se puede observar que a pesar de la predominante introducción de las TIC en los centros educativos, en este espacio sigue sin haber sitio para las familias. Para superar esta desconexión digital entre escuela y familias los autores señalan la necesidad de convertir estas herramientas en espacios de comunicación y participación real, construidas a partir del trabajo conjunto de todos los agentes implicados.

En este mismo año, Vallespir, Rincón y Morey (2016), analizan la participación de las familias en el Consejo Escolar, así como la formación del profesorado sobre esta cuestión. El objetivo primordial era identificar y analizar factores que inciden en la participación de las familias en los Consejos Escolares a partir de la información de los docentes y de las propias familias. Para esto, la investigación consistió en una serie de entrevistas en profundidad a ambos colectivos. A partir del análisis de contenido de dichas entrevistas, los resultados se agruparon en un conjunto de factores para cada colectivo. En el caso de las familias destaca que los propios padres y madres denuncian la falta de participación así como un gran desconocimiento del funcionamiento y de la utilidad del Consejo Escolar. Al mismo tiempo consideran que el profesorado tiene una excesiva representación en este órgano, con la consiguiente minoría de las familias, lo que les lleva a tener poco poder de decisión. En cuanto al colectivo docente, este señala que los centros educativos siempre impulsaron la participación de las familias pero que, sin embargo, hay un gran desconocimiento por parte de éstas acerca de la utilidad del Consejo Escolar u otras formas de participación. Además señalan la pérdida de poder que experimentó el Consejo Escolar con la LOMCE, así como la excesiva burocratización y la composición desigual. Analizando estos resultados se puede observar que ambos colectivos entrevistados mostraron puntos en común en sus percepciones, como por ejemplo, los bajos niveles de participación y el desconocimiento de la existencia y del funcionamiento del Consejo Escolar. Por lo tanto, resulta necesario llevar a cabo acciones de formación en el ámbito de la participación tanto para el colectivo docente como para el colectivo de las familias.

\section{Expectativas y opiniones de la familia y del profesorado}

Para tratar de dar respuesta al objetivo de analizar la situación existente en cuanto a las relaciones entre las familias y la escuela, es importante conocer las expectativas y las opiniones que tienen al respecto ambos colectivos implicados. De este modo, García- 
Bacete (2006) realizó un estudio con el objetivo de conocer la opinión del profesorado sobre las relaciones entre los centros educativos y las familias del alumnado allí escolarizado. Dado que la principal variable a tener en cuenta en esta investigación era la opinión del profesorado, el instrumento de evaluación empleado fue un cuestionario compuesto por 125 preguntas (65 abiertas y 60 cerradas), que hacían referencia a cuatro grandes apartados: Acción Tutorial, El tutor, Relaciones Escuela-Familia, y Reuniones de tutoría con los padres. Sin embargo, para el estudio solo se analizaron las respuestas referidas al grupo de las Relaciones Escuela-Familia. Por su parte, las variables que se tuvieron en cuenta con respecto a los centros, a los profesores y a las familias fueron las siguientes: localización del centro, tamaño del centro, nivel sociocultural de las familias, género del profesorado, edad del profesorado, etapa educativa en la que ejerce la labor docente, experiencia del profesorado, antigüedad en un mismo centro, y titulación académica. Los resultados evidencian que, en cuanto al papel que los docentes consideran que desempeñan los padres en la escuela, las respuestas fueron muy diversas. Aún así, la categoría más predominante fue la denominada como "Centrada en el Centro", seguida de "Centrada en el hijo". En lo referente a la satisfacción con las relaciones escuela-familia un $60 \%$ del profesorado cualifica dichas relaciones como óptimas y positivas, mientras que un $25 \%$ las define como negativas y mejorables. La siguiente dimensión analizada hacía referencia a cómo eran esas relaciones en la realidad y se observó que la mayoría de las relaciones son de carácter presencial, tanto desde las escuelas a las familias como al revés. Solo un $28 \%$ de los docentes emplea además otros medios como pueden ser escritos o el teléfono. El tutor es el elemento clave de esas relaciones, siendo el principal interlocutor entre los dos sistemas implicados. Por su parte, las respuestas sobre como deberían cambiar las relaciones de la escuela con las familia y viceversa indican que la mayoría del profesorado sitúa esos elementos de cambio bien en las familias, bien en el centro, pero muy pocos en la propia labor docente. Concretamente, un $35,90 \%$ del profesorado se mostraban satisfecho con su desempeño profesional. Por último, las principales dificultades que los docentes encontraron para que realmente existan las deseables relaciones entre familia y escuela son las siguientes: falta de tiempo por parte de la familia, dificultades de comunicación entre profesores y familias, falta de interés de las familias por la educación de sus hijos, condiciones de vida o problemas familiares, desacuerdo de roles y falta de participación debido a la escasa tradición por parte de ambos agente implicados para favorecer la participación en la educación escolar. Por lo tanto, en relación con nuestro primer objetivo, con este estudio se pone en evidencia que las expectativas que los profesores tienen sobre esta relación o bien son bajas porque ya se muestran satisfechos o bien sitúan el foco de cambio en las familias casi exclusivamente, demandando que las relaciones de éstas con el centro sean más frecuentes, fluidas y respetuosas.

En su artículo de 2006, Hernández y López reflexionan sobre la situación actual de la relación entre familias y escuela y concluyen que las familias depositan sobre el sistema educativo las siguientes expectativas: un sistema educativo de calidad que cuente con la financiación necesaria para poder cubrir todas las necesidades materiales y profesionales precisadas por el alumnado; un sistema educativo competente a nivel europeo en lo referente a la capacitación técnica, disminuyendo así el fracaso escolar; que las escuelas preparen a los alumnos para un desarrollo profesional, ofreciendo garantías de poder entrar en el mercado laboral; una escuela en la que se lleve a cabo un trabajo en equipo del profesorado; un seguimiento más individualizado de cada alumno y una mayor labor de orientación educativa; que se revisen los contenidos curriculares para orientarlos hacia la formación de ciudadanos cultos y críticos; revisión de las metodologías; mayor formación del profesorado; y un sistema educativo flexible y adaptado a los cambios sociales y familiares existentes. 
Por último, también es importante analizar la formación que reciben los futuros docentes en este tema, ya que ese es un elemento fundamental para la mejora de las relaciones entre familia y escuela. En este sentido, Gomilla y Pascual (2015), realizaron una investigación para analizar la percepción del profesorado en formación sobre la participación de las familias en los centros educativos. Para eso, estos autores llevaron a cabo un análisis cuantitativo y cualitativo de las experiencias y opiniones manifestadas por el profesorado de primaria en formación en el último curso de Magisterio del plan antiguo. Los instrumentos empleados fueron un cuestionario antes del inicio de curso y grupos de discusión con el alumnado al finalizar el último período de prácticas externas en los centros educativos. Los resultados indicaron, en relación con nuestro primer objetivo, que la opinión mayoritaria era que las familias deben participar en los centros educativos, principalmente a través de las tutorías, de la AMPA o de la coordinación con el profesorado. En cuanto a las barreras que dificultan la participación, los futuros docentes señalaron el desconocimiento de los canales de participación, la disponibilidad de las familias y el interés de las mismas. A estas opiniones se suma la escasez de intervenciones con las familias durante la realización del período de prácticas en el centro educativo, por lo que los alumnos de Magisterio acaban asumiendo que los padres participan poco y se lo hacen es exclusivamente en conflictos o fiestas/eventos. En lo referente a la etapa de Educación Secundaria, no se conocen estudios equivalentes.

Tabla 1.

Principales Estudios y Resultados de las Relaciones Familia-Escuela

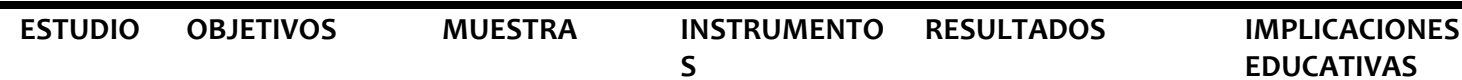

\begin{tabular}{|c|c|c|c|c|c|}
\hline $\begin{array}{l}\text { García- } \\
\text { Bacete } \\
(2006)\end{array}$ & $\begin{array}{l}\text { Conocer la } \\
\text { opinión del } \\
\text { profesorado } \\
\text { sobre las } \\
\text { relaciones entre } \\
\text { escuelas y } \\
\text { familias. }\end{array}$ & $\begin{array}{l}\text { N: } 100 \\
\text { docentes de } \\
\text { E.I. y E.P. }\end{array}$ & Cuestionario & $\begin{array}{l}\text { La mayoría del } \\
\text { profesorado se } \\
\text { muestra satisfecho } \\
\text { con las relaciones } \\
\text { existentes y } \\
\text { considera que el } \\
\text { cambio debe } \\
\text { proceder de la } \\
\text { actitud de las } \\
\text { familias. }\end{array}$ & $\begin{array}{l}\text { Más formación al } \\
\text { profesorado sobre } \\
\text { las relaciones con } \\
\text { las familias para } \\
\text { conseguir un } \\
\text { cambio de actitud } \\
\text { de los mismos. }\end{array}$ \\
\hline
\end{tabular}

\begin{tabular}{|c|c|c|c|c|c|}
\hline $\begin{array}{l}\text { Hernánd } \\
\text { ez y } \\
\text { López } \\
(2006)\end{array}$ & $\begin{array}{l}\text { Conocer las } \\
\text { expectativas } \\
\text { que los padres } \\
\text { depositan en la } \\
\text { escuela. }\end{array}$ & $\begin{array}{l}\text { Información } \\
\text { procedente } \\
\text { del } n^{\circ} 80 \text { de la } \\
\text { Revista de } \\
\text { padres y } \\
\text { madres de } \\
\text { alumnos } \\
\text { (CEAPA, 2004). }\end{array}$ & $\begin{array}{l}\text { Información } \\
\text { procedente del } \\
n^{\circ} 80 \text { de la } \\
\text { Revista de } \\
\text { padres y } \\
\text { madres de } \\
\text { alumnos } \\
\text { (CEAPA, 2004). }\end{array}$ & $\begin{array}{l}\text { Las familias } \\
\text { depositan en las } \\
\text { escuelas las } \\
\text { siguientes } \\
\text { expectativas: calidad } \\
\text { educativa, cambios } \\
\text { curriculares y } \\
\text { metodológicos, y } \\
\text { mayor formación de } \\
\text { los profesionales } \\
\text { educativos. }\end{array}$ & $\begin{array}{l}\text { Tener en cuenta } \\
\text { las expectativas de } \\
\text { las familias a la } \\
\text { hora de planificar } \\
\text { las acciones } \\
\text { educativas, } \\
\text { fomentando así } \\
\text { una mejora de las } \\
\text { relaciones entre } \\
\text { ambos colectivos. }\end{array}$ \\
\hline $\begin{array}{l}\text { Blândul } \\
\text { (2012) }\end{array}$ & $\begin{array}{l}\text { Investigar las } \\
\text { relaciones } \\
\text { existentes entre } \\
\text { familia y } \\
\text { escuela. }\end{array}$ & $\begin{array}{l}\mathrm{N}: 60 \text { docentes } \\
\text { de } 5 \text { centros } \\
\text { rurales. }\end{array}$ & Cuestionario. & $\begin{array}{l}\text { Existencia de un } \\
\text { patrón de } \\
\text { comunicación } \\
\text { unidireccional: desde } \\
\text { el profesorado hacia }\end{array}$ & $\begin{array}{l}\text { Necesidad de } \\
\text { canales de } \\
\text { comunicación y } \\
\text { participación de } \\
\text { carácter }\end{array}$ \\
\hline
\end{tabular}




\begin{tabular}{ll}
\hline Gomilla y & Analizar la \\
Pascual & percepción del \\
(2015) & profesorado en \\
& formación sobre \\
& la participación \\
& de las familias \\
& en los centros \\
& educativos.
\end{tabular}

\begin{tabular}{llll} 
& & las familias. & bidireccional. \\
\hline $\begin{array}{lll}\text { Profesorado } \\
\text { en formación, }\end{array}$ & Cuestionario & Consideración & Necesidad de \\
último curso & del curso. & mayoritaria de que & mejorar la \\
de Magisterio. & las familias deben & formación del \\
& Grupos de & participar en los & profesorado, \\
& discusión al & centros, pero & introduciendo más \\
& terminar las & escasez de & contenidos \\
& prácticas & intervenciones con & específicos sobre \\
& externas. & las familias durante & las familias.
\end{tabular}

el período de prácticas de los futuros docentes.

\begin{tabular}{|c|c|c|c|c|c|}
\hline $\begin{array}{l}\text { Kim y Hill } \\
(2015)\end{array}$ & $\begin{array}{l}\text { Estudiar la } \\
\text { influencia de la } \\
\text { participación de } \\
\text { las familias en la } \\
\text { escuela sobre el } \\
\text { rendimiento } \\
\text { académico del } \\
\text { alumnado y } \\
\text { analizar } \\
\text { diferencias } \\
\text { entre padres y } \\
\text { madres. }\end{array}$ & $\begin{array}{l}\text { Metaanálisis } \\
\text { con } 52 \\
\text { estudios } \\
\text { empíricos. }\end{array}$ & & $\begin{array}{l}\text { Correlación } \\
\text { significativa y } \\
\text { positiva entre } \\
\text { participación familiar } \\
\text { y rendimiento } \\
\text { académico, sin } \\
\text { diferencia de género } \\
\text { entre padres y } \\
\text { madres. }\end{array}$ & $\begin{array}{l}\text { Diseñar } \\
\text { estrategias que } \\
\text { fomenten la } \\
\text { participación } \\
\text { familiar y que } \\
\text { repercutan } \\
\text { positivamente a } \\
\text { nivel académico. }\end{array}$ \\
\hline $\begin{array}{l}\text { Hampde } \\
\mathrm{n} \text { - } \\
\text { Thompso } \\
\mathrm{n} \text { y } \\
\text { Galindo } \\
(2016)\end{array}$ & $\begin{array}{l}\text { Estudiar la } \\
\text { relación entre } \\
\text { escuela y familia } \\
\text { y su asociación } \\
\text { con el } \\
\text { rendimiento } \\
\text { académico. }\end{array}$ & $\begin{array}{l}\mathrm{N}: 10.572 \\
\text { adolescentes. } \\
\text { Edad: 13-16 } \\
\text { años. }\end{array}$ & $\begin{array}{l}\text { Datos } \\
\text { extraídos de un } \\
\text { estudio } \\
\text { longitudinal } \\
\text { realizado a } \\
\text { adolescentes } \\
\text { en Inglaterra. }\end{array}$ & $\begin{array}{l}\text { Relación significativa } \\
\text { entre participación } \\
\text { de la familia en la } \\
\text { escuela y } \\
\text { rendimiento } \\
\text { académico, mediada } \\
\text { por la satisfacción } \\
\text { escolar de los } \\
\text { padres. }\end{array}$ & $\begin{array}{l}\text { Empezar por } \\
\text { mejorar la } \\
\text { satisfacción de las } \\
\text { familias para que } \\
\text { esto suponga un } \\
\text { incremento en la } \\
\text { participación. }\end{array}$ \\
\hline $\begin{array}{l}\text { Seoane y } \\
\text { Sabé } \\
(2016)\end{array}$ & $\begin{array}{l}\text { Analizar las } \\
\text { relaciones } \\
\text { digitales entre } \\
\text { profesorado y } \\
\text { familias. }\end{array}$ & $\begin{array}{l}\mathrm{N}: 10 \text { centros } \\
\text { educativos. }\end{array}$ & $\begin{array}{l}\text { Análisis de } \\
\text { documentos. } \\
\text { Entrevistas } \\
\text { semiestructura } \\
\text { das. }\end{array}$ & $\begin{array}{l}\text { La introducción de } \\
\text { las TIC no supuso } \\
\text { cambios importantes } \\
\text { en los contenidos ni } \\
\text { en la forma de } \\
\text { relación familia- } \\
\text { escuela. }\end{array}$ & $\begin{array}{l}\text { Introducir las TIC } \\
\text { como un espacio } \\
\text { para la } \\
\text { comunicación y la } \\
\text { participación, no } \\
\text { solo como un } \\
\text { instrumento } \\
\text { informativo. }\end{array}$ \\
\hline $\begin{array}{l}\text { Vallespir, } \\
\text { Rincón y } \\
\text { Morey } \\
\text { (2016) }\end{array}$ & $\begin{array}{l}\text { Analizar la } \\
\text { participación de } \\
\text { las familias en } \\
\text { los Consejos } \\
\text { Escolares y la } \\
\text { formación del } \\
\text { profesorado } \\
\text { sobre este } \\
\text { tema. }\end{array}$ & $\begin{array}{l}\text { N: } 260 \text { sujetos } \\
\text { (138 docentes } \\
\text { y } 122 \\
\text { miembros de } \\
\text { familias). }\end{array}$ & $\begin{array}{l}\text { Entrevista a } \\
\text { profesorado y } \\
\text { familias. }\end{array}$ & $\begin{array}{l}\text { La participación de } \\
\text { las familias en el } \\
\text { Consejo Escolar es } \\
\text { valorada como } \\
\text { positiva pero, sin } \\
\text { embargo, no es real } \\
\text { ni efectiva. }\end{array}$ & $\begin{array}{l}\text { Más información y } \\
\text { formación a las } \\
\text { familias sobre } \\
\text { formas de } \\
\text { participación en el } \\
\text { centro educativo. }\end{array}$ \\
\hline
\end{tabular}

\section{Discusión y conclusiones}

Si hacemos un poco de historia reciente, todas las normativas existentes en materia de educación (LGE, LOGSE, LOE, LOMCE) han resaltado y resaltan la necesidad de establecer relaciones positivas y efectivas entre los centros escolares y las familias, a la vez que 
promover la implicación de estas en la educación de sus hijos. Sin embargo, en la realidad de la práctica educativa se observa que esta relación si se da es entre las familias y el profesor tutor y, en la mayoría de los casos, son contactos precedidos por situaciones problemáticas de carácter académico o comportamental (Martínez González y Pérez Herrero, 2006). Además, la mayoría del profesorado se muestra satisfecho con su actuación y propone que el cambio debe proceder de las familias. Por lo tanto, resulta necesario contar con una figura en el centro educativo que cuente con la necesaria formación tanto para dinamizar al profesorado como para motivar y atraer el interés de las familias, y esta figura puede ser el orientador. Así, en relación con el objetivo de realizar una serie de aportaciones prácticas para el Departamento de Orientación que permitan mejorar las relaciones familia-escuela, dicho profesional, actuando con un rol de mediador, asesor y dinamizador debería seguir las siguientes acciones prioritarias para promover la cooperación entre las familias, los centros, y distintas entidades comunitarias: incrementar la frecuencia de comunicación entre la familia y el profesorado para consensuar los objetivos educativos a seguir con los menores; establecer más contactos entre ambos colectivos para informar no solo de situaciones problemáticas, sino también de aspectos positivos en lo que respecta al progreso de los adolescentes; poner en común con más frecuencia las inquietudes que tanto profesorado como padres y madres puedan tener en lo relacionado con la educación de sus hijos; incrementar acciones que mejoren la competencias de las familias en el desarrollo de las funciones educativas; incrementar el número de actividades realizadas en el centro abiertas a la participación de las familias; y proporcionar formación y asesoramiento al profesorado para mejorar su capacidad para trabajar colaborativamente con las familias (Martínez González y Pérez Herrero, 2006).

La mejora de las relaciones de los adolescentes con sus familias, así como de las familias con la escuela, encuentra en los profesionales de la orientación existentes en los centros educativos agentes idóneos para llevarla a cabo. Sin embargo, aunque las acciones deben ser impulsadas por estos profesionales, es necesaria la implicación de todos los miembros de la comunidad educativa, ya que es preciso trabajar desde una perspectiva sistémica.

Teniendo en cuenta que una de las vías de participación que existen actualmente para las familias son las AMPAs, se debe promover y permitir que éstas desarrollen sus actividades integradas en el currículo escolar del alumnado. Existen experiencias ya de algunas AMPAS que trabajan en las aulas con talleres de educación musical, educación para la salud, interculturalidad, informática, etc. También sería recomendable promover la formación de las familias, proporcionando conocimientos e información en sesiones grupales, similares a lo que tradicionalmente se conoce como Escuelas de Padres o Escuelas de Familias, pero siempre partiendo de los intereses y de las demandas de los destinatarios. Los profesionales de la orientación pueden aprovechar estas sesiones para dar a conocer los distintos Planes y Documentos existentes en el centro, pues el primer paso para implicarse y participar en la vida escolar es conocer la organización y el funcionamiento del centro educativo. Estas Escuelas de Familias deben contar también con la colaboración del profesorado del centro, para que conjuntamente con las familias puedan analizar las actuaciones existentes y decidir otras que permitan mejorar el bienestar y el rendimiento de los alumnos (Ramírez, García y Sánchez, 2011). Además, también pueden contar con delegados de padres y madres, que representen a este colectivo ante el centro y que tengan la función de transmitir sus inquietudes y sus propuestas de mejora o de intervención (Coronado, 2011).

Independientemente de las acciones puntuales que se puedan llevar a cabo, existe un amplio consenso en señalar que la mejora educativa pasa por la apertura del centro educativo a la comunidad. Si tenemos en cuenta los resultados del Proyecto INCLUD-ED 
(2011), en relación con nuestro segundo objetivo, solo tres formas de participación denominadas como decisiva, evaluativa y educativa, tienen un impacto positivo en el aprendizaje del alumnado y en la promoción de la mejora colectiva de la comunidad, generando un mayor compromiso e implicación de las familias en las escuelas. Las restantes formas de participación (informativa y consultiva), que son las que mayoritariamente se llevan a cabo en los centros educativos, no contribuyen a estos resultados. La participación decisiva, evaluativa y educativa son las que se llevan a cabo en las Comunidades de Aprendizaje en las que tanto familias como demás miembros de la comunidad intervienen en las aulas junto con el profesorado para contribuir al aprendizaje del alumnado. En este tipo de escuelas, la base del aprendizaje es la comunicación y la interacción entre distintas personas de la comunidad, de las cuales todas tienen algo que aportar a los demás. Son frecuentes los grupos interactivos, grupos heterogéneos de alumnos que trabajan colaborativamente en la realización de una tarea supervisados por un adulto, el cual puede ser un profesor, un familiar, un voluntario, etc. Además todos los miembros participan en la toma de decisiones educativas mediante la reflexión y el diálogo.

A pesar de que en todos los estudios presentados en este trabajo la tendencia es señalar que la participación de la familia en la escuela no es ni real ni efectiva, dichos estudios no están ausentes de limitaciones que condicionan los resultados propuestos. La mayoría de las investigaciones son de carácter transversal cuando, lo ideal es que sean longitudinales para que realmente permitan establecer relaciones de causalidad entre los fenómenos estudiados. Esto dificulta la generalización de los resultados. Muchos de los estudios también presentan como limitación que se centran en información aportada exclusivamente por uno de los colectivos (familia o escuela), sin embargo, para un mayor rigor y fiabilidad de los resultados, la información debe proceder de todos los agentes implicados ya que cada uno de ellos tendrá su punto de vista.

\section{Referencias}

Arnett, J. J. (2008). Adolescencia y adultez emergente: un enfoque cultural. México: Pearson Educación.

Blândul, V. C. (2012). The partnership between school and family-cooperation or conflict?. Procedia-Social and Behavioral Sciences, 47, 1501-1505.

Coronado, A. (2011). La Orientación y Tutoría a Familias desde un modelo inclusivo y ecosistémico. I Jornadas Andaluzas de Orientación Educativa. Málaga: Universidad de Málaga.

Elzo, J. (2006). La educación familiar en un mundo en cambio. En Educación y familia: la educación familiar en un mundo en cambio (pp. 1-21). Universidad Pontificia Comillas.

García-Bacete, F. J. (2006). Cómo son y cómo podrían ser las relaciones entre escuelas y familias en opinión del profesorado. Cultura y educación, 18(3-4), 247-265.

Gomilla, M.A. y Pascual, B. (2015). La participación de las familias en el sistema educativo: la percepción del profesorado en formación. Revista Electrónica Interuniversitaria de Formación del Profesorado, 18(3), 99-112.

Hampden-Thompson, G., y Galindo, C. (2016). School-family relationships, school satisfaction and the academic achievement of young people. Educational Review, 118. 
Hernández, M. Á. y López, H. (2006). Análisis del enfoque actual de la cooperación padres y escuela. Aula abierta, (87), 3-25.

INCLUD-ED (2011). Actuaciones de éxito en las escuelas europeas. Madrid: Ministerio de Educación, Cultura y Deporte. Subdirección General de Documentación y Publicaciones.

Kim, S. W., y Hill, N. E. (2015). Including fathers in the picture: A meta-analysis of parental involvement and students' academic achievement. Journal of Educational Psychology, 107(4), 919-934.

Martínez-González, R. A., y Pérez-Herrero, M. H. (2006). Propuestas metodológicas para una educación de calidad a través de las relaciones entre centros docentes, familias y entidades comunitarias. Cultura y Educación, 18(3-4), 231-246.

Oliva, A. (2003). Adolescencia en España a principios del siglo XXI. Cultura y educación, 15(4), $373-383$.

Oliva, A. (2006). Ideas y expectativas sobre educación en la adolescencia: familia y adolescencia en una sociedad en cambio. En M. I. Álvarez e A. Berástegui, Educación y familia: la educación familiar en un mundo en cambio (pp. 65-82). Universidad Pontificia Comillas.

Ramírez, S., García, A., y Sánchez, C. A. (2011). El éxito escolar: cómo pueden contribuir las familias del alumnado?. Confederación Española de Asociaciones de Padres y Madres de Alumnos.

Seoane, M. B., y Sabé, J. C. (2016). Las relaciones digitales entre familias y escuela: análisis y propuestas. @ tic. revista d'innovació educativa, (16), 1-9.

Siles Rojas, C. (2003). La colaboración de los padres con la escuela. Revista Padres y Maestros, no. 279, pp. 10-14.

Vallespir, J., Rincón, J.C. y Morey, M. (2016). La participación de las familias en el Consejo Escolar y la formación del profesorado. Revista Electrónica Interuniversitaria de Formación del Profesorado, 19 (1), 31-45. 